\title{
Deep-Inelastic Electroproduction of Neutrons in the Proton Fragmentation Region
}

\author{
Boris Kopeliovich', Bogdan Povh \\ Max-Planck Institut für Kernphysik, Postfach 103980, \\ 69029 Heidelberg, Germany \\ and \\ Irina Potashnikova \\ Joint Institute for Nuclear Research, Laboratory of Nuclear Problems, \\ Dubna, 141980 Moscow Region, Russia
}

\begin{abstract}
Experiments at HERA looking for deep-inelastic electroproduction of neutrons in the proton fragmentation region are in progress. They are aimed to measure the pion structure function at small Bjorken $x$. The important condition for such a study is to establish under what kinematical conditions the dominance of the pion-pole graph in the process is guaranteed. We analyse other sources of the leading neutron, in order to figure out the kinematical region where the one-pion exchange dominates.
\end{abstract}

* On leave from Joint Institute for Nuclear Research, Laboratory of Nuclear Problems, Dubna, 141980 Moscow Region, Russia. E-mail: bzk@dxnhd1.mpi-hd.mpg.de 


\section{Introduction}

Deep-inelastic lepton scattering off a proton at small Bjorken $x$ probes the distribution of sea quarks and antiquarks in the proton. The cloud of virtual pions surrounding the nucleon core of the proton is known to be an important source of the sea quarks [1]. Thus, one may hope to be able to extract information about the pion structure function from such measurements, relying upon the dominance of the one-pion-exchange diagram shown in fig. 田a [2, 目, 团.

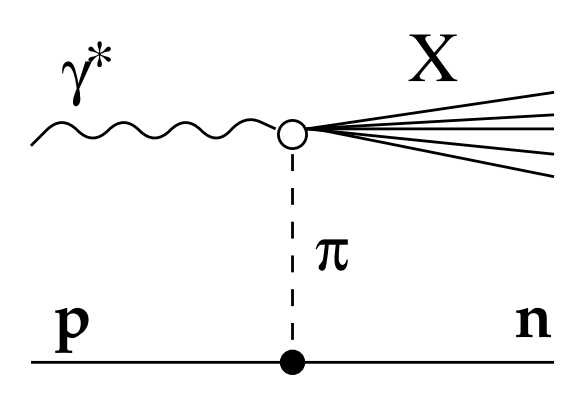

a

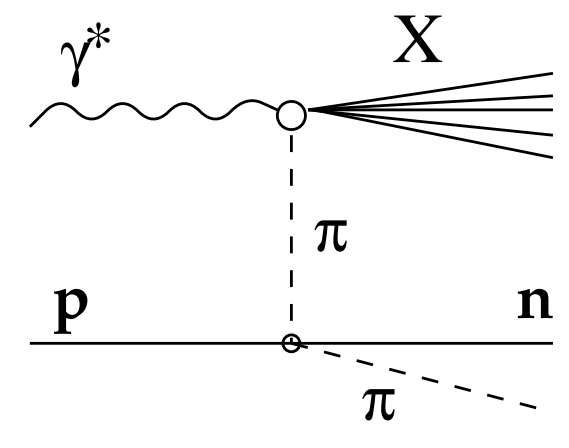

b

Figure 1: One-pion-exchange diagrams contributing to the deep-inelastic scattering $e p \rightarrow e^{\prime} n X$ without (a) and with (b) production of an additional pion in the proton fragmentation region.

One has to provide, however, convincing evidence that the sea quarks probed by the virtual photons do really belong to the pion. There are many other sources of sea quarks the contribution of which has to be evaluated reliably. Moreover, even if one is sure that the probed sea quark originates from a pion, in order to extract the pion structure function from the data, one relies upon the diagram in fig. 17a. Another one depicted in fig. 罥 (as well as others with additional particle production in the pion-proton interaction vertex) should be considered as an unwanted background, because they result in a different relation between the deep-inelastic scattering cross section and the pion structure functions.

In the present paper we study the background mechanisms of the leading neutron production in deep-inelastic scattering, which may distort the determination of the pion structure function. We conclude that there is a possibility to suppress the backgrounds if the neutron 
is detected within $z$-interval $0.7-0.9$ and at $|t|<0.2-0.3 \mathrm{GeV}^{2}$.

Although the recommendations of the previous analysis [2]-[四 are not very far from our conclusion, our results are quite different at some essential points concerning the pion pole contribution, as well as that for the background. Furthermore, as the diagram in fig. 1 $1 \mathrm{~b}$ cannot be evaluated using the light-cone representation for the nucleon wave function, we therefore apply the standard Feynman diagram technique.

\section{The signatures of the pion}

What is specific about the pion, which may help to single out its contribution as compared to other mesons surrounding the proton? Of course it is the smallness of the pion mass. It allows the pion to be spread around the proton at much longer distances than other mesons. This fact manifests itself as a narrow transverse-momentum distribution, $\left\langle p_{T}^{2}\right\rangle \sim m_{\pi}^{2}$. The same argument is applicable to the diagram in fig. 1 $\mathrm{b}$, but the narrow peak in the $p_{T}$ distribution of the neutrons from the process in fig. 19a will be smeared out by the emission of the additional pion, which depends on the effective mass of the final $\pi n$ system.

Thus, an observation of a narrow peak in the $p_{t}$-distribution of the produced neutrons would serve as a solid evidence of the pion-exchange dominance.

The above arguments, however, fail if the pion is far off the mass shell. This depends on the energy $\nu$ of the virtual photon and the effective mass $M$ of the produced jet. Indeed, if the pion in the rest frame of the proton has a "mass" $\sqrt{|t|}$, where $t<0$ is the pion fourmomentum squares, the photon cannot produce a jet, with an effective mass larger than $M^{2} \approx 2 \nu \sqrt{|t|}$. Thus, the production of a heavy mass jet, $\left(M^{2} / 2 \nu\right)^{2} \gg m_{\pi}^{2}$ needs a highly virtual pion target, $|t| \gg m_{\pi}^{2}$, and the width of $p_{T}^{2}$ distribution of the produced neutrons is determined by $|t|$ rather than by $m_{\pi}^{2}$. This means that such a far-off-mass-shell pion does not propagate far away from the proton, but only for a short distance of about $1 / \sqrt{|t|}$. This area is overpopulated by sea quarks having other than a pion origin, i.e. the pion loses its signature.

In order to make $|t|$ sufficiently small and single out the pion contribution one may 
select the events with a small value of $\left(M^{2} / 2 \nu\right)^{2} \leq m_{\pi}^{2}$. However, in this case one faces another problem, which is related to the spinelessness of the pion. It is true that the pion contribution is enhanced at large transverse distances from the proton, what corresponds to small $\left\langle p_{T}^{2}\right\rangle$ of the recoil neutron. However, the rapidity gap covered by the virtual pion becomes large in this case, $\Delta y \approx \ln (1-z)$, where $1-z \approx M^{2} / 2 m_{N} \nu \leq m_{\pi} / m_{N}$. We know that the energy dependence of the scattering amplitude corresponding to a particle exchange is controlled by the spin of the particle. In the Regge theory the Reggeon intercept plays the role of the spin. It is about the same as the spin for the pion, $\alpha_{\pi}(0) \approx 0$; is higher for leading Reggeons $\rho$ and $a_{2}, \alpha_{R}(0) \approx 1 / 2$; and it has the highest value for the Pomeron, $\alpha_{P}>1$. Therefore, the probability for a pion to cover a large rapidity gap is suppressed by the factor $\exp (-2 \Delta y)$, which is smaller than the corresponding factor $\exp (-\Delta y)$ for the leading Reggeons $\rho$ and $a_{2}$ (see below), or for the Pomeron, which is rapidity-independent, or even grows with $\Delta y$. In such circumstances one should be very cautious about the competing mechanisms of generation of the sea quarks. Examples of diagrams corresponding to the contribution of these mechanisms to the neutron production are shown in fig. 2.

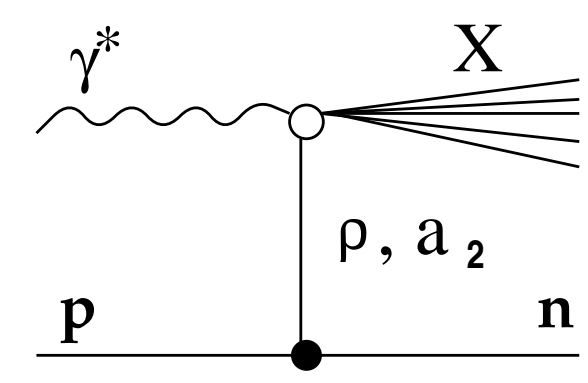

a

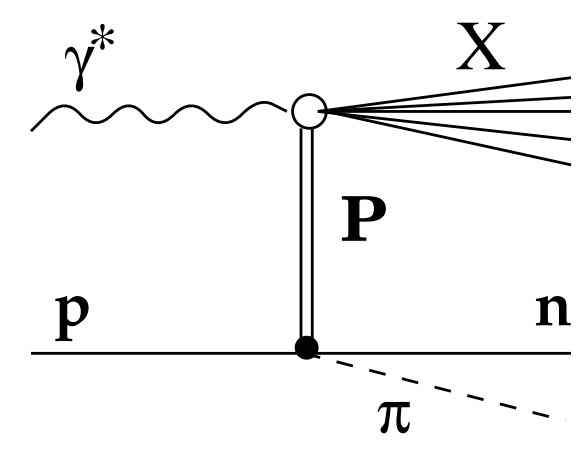

b

Figure 2: The $\rho$ - and $a_{2}$-Reggeon (a) and the Pomeron (b) exchange contributions to the deep-inelastic scattering $e p \rightarrow e^{\prime} n X$.

We conclude that any attempts to single out the pion-pole contribution relying upon the signatures of the pion, the smallness of its mass and spinelessness, face kinematical restrictions which in some sense exclude each other. In such circumstances one should look for a compromise, i.e. a kinematical region where the pion pole nevertheless dominates. 
This is the goal of the present paper.

\section{The pion-pole contribution to $e p \rightarrow e^{\prime} n X$ (fig. 1a)}

First of all, we should calculate the diagram shown in fig. 1a, which is to be dominant in order to extract the pion structure function from data on the reaction $e p \rightarrow e^{\prime} n X$. The corresponding cross section is well known [5] and reads

$$
\left[\frac{d F_{2}^{p \rightarrow n}\left(x, Q^{2}\right)}{d p_{T}^{2} d z}\right]_{1 a}=\frac{2 g_{\pi}^{2}}{16 \pi^{2}} \frac{|t|}{\left(m_{\pi}^{2}-t\right)^{2}} G_{1}^{2}(t)(1-z)^{1+2 \alpha_{\pi}^{\prime}|t|} F_{2}^{\pi}\left(x_{\pi}, Q^{2}\right) .
$$

We use the following notations: $\vec{p}_{T}$ is the transverse component of the neutron momentum relative to the direction of the virtual photon; $z$ is the Lorenz-invariant ratio of the neutron to proton light-cone momenta. The semi-inclusive structure function $F_{2}^{p \rightarrow n}\left(x, Q^{2}\right)$ is related to the cross section of electroproduction of the neutron,

$$
\left[\frac{d \sigma\left(e p \rightarrow e^{\prime} n X\right)}{d p_{T}^{2} d z d x d Q^{2}}\right]_{1 a}=\frac{2 \pi \alpha_{e m}^{2}}{x Q^{4}}\left(2-2 y+y^{2}\right) \frac{d F_{2}^{p \rightarrow n}\left(x, Q^{2}\right)}{d p_{T}^{2} d z}
$$

where $y=Q^{2} / x s ; s$ is the c.m. $\gamma^{*} p$ total energy squared. We neglect the small contribution to the cross section of the longitudinally polarized photons. The Bjorken variable for the pion is

$$
x_{\pi}=\frac{x_{p}}{1-z-x_{p}},
$$

The $\pi^{0} N N$ coupling $g_{\pi}$ is fixed at $g_{\pi}^{2} / 4 \pi=13.75$ [6, 7].

The pion four-momentum squared is related to the observables as

$$
t=-\frac{1}{z}\left[p_{T}^{2}+(1-z)^{2} m_{N}^{2}\right]
$$

The common $t$-dependence of the formfactor for the pion-nucleon vertex and the virtual pion photoabsorption cross section is effectively parameterized in the form, $G_{1}(t)=$ $\exp \left[R_{1}^{2}\left(t-m_{\pi}^{2}\right)\right]$, where the slope $R_{1}^{2}$ is to be fitted to experimental data on reactions dominated by pion exchange. Unfortunately the results of such an analysis [8, 9, 1, 10, 11] are not stable, and $R_{1}^{2}$ varies from zero (see in [3]) up to $2 \mathrm{GeV}^{-2}$ [8]. We fix $R_{1}^{2}=0.3 \mathrm{GeV}^{-2}$ according to the results of [9, 1, 10] which seem to us most reliable. 
It should be pointed out that the formfactor used in [4] has a different form, namely $G_{1}=\exp \left[R^{2} t /(1-z)\right]$. This formfactor was introduced ad hoc in [12] and has no justification experimentally (compare the descriptions of the data on $\Delta$ production at small $(1-z)$ in refs. [12] and [13]), and gives energy-dependent $R_{1}^{2}$, which contradicts the results of the approach based on Feynman diagrams, leading to an abnormal growth of the effective radius at $z \rightarrow 1$.

We take into account the Reggeization of the pion exchange in eq. (1), this is why we have the term $2 \alpha_{\pi}^{\prime} t$ as a power of $(1-z)$, where $\alpha_{\pi}^{\prime} \approx 1 \mathrm{GeV}^{-2}$ is the slope of the pion Regge trajectory. As this is different from the statement in [4], the Reggeization is important in the kinematical region under discussion. The term $2 \alpha_{\pi}^{\prime}$ in the effective slope of $p_{T}^{2}$-distribution contributes more than the formfactor radius $R_{1}^{2}$.

In the experiment $F_{2}^{\pi}\left(x, Q^{2}\right)$ will be determined by a fit to experimental data on electroproduction of the neutron. In order to obtain a numerical estimate of the cross sections we assume $x_{\pi} \ll 1$. Thinking in terms of the constituent quark model, one may expect a simple relation $F_{2}^{\pi}=2 / 3 F_{2}^{p}$. The same relation is also predicted by the low-order perturbative QCD calculations [14. We use for $F_{2}^{p}\left(x, Q^{2}\right)$ the results of the fit 15 to low- $x$ data. We checked that another parameterization from [16] does not produce any visible distinction.

Our predictions for $z$ - and $p_{T}^{2}$-dependence of the cross section of inclusive electroproduction of neutrons at $Q^{2}=10 \mathrm{GeV}^{2}$ and $x_{p}=5 \cdot 10^{-4}$ is depicted in fig. 3 .

We see that the cross section steeply vanishes towards $z=1$. This is due to the factor $(1-z)$ in eq. (1), which originates from the spinelessness of the pion according to the discussion in the previous section. Small $(1-z)$ correspond to large rapidity gaps, which provide a strong suppression of any Regge exchanges with low intercepts.

\section{The pion-pole contribution to $e p \rightarrow e^{\prime} n \pi X$ (fig. 1b)}

As a result of one-pion exchange the proton can be excited, what ends up with production of extra particles. This contribution was never evaluated, except for the production of the $\Delta(1236)$ resonance. The simplest and probably most important case is the production of an additional pion, as is shown in fig. 1 $\mathrm{b}$. In this case we have to replace the $\pi N N$ vertex by the $\pi p \rightarrow \pi n$ charge-exchange scattering amplitude. This amplitude, if it is a realistic 


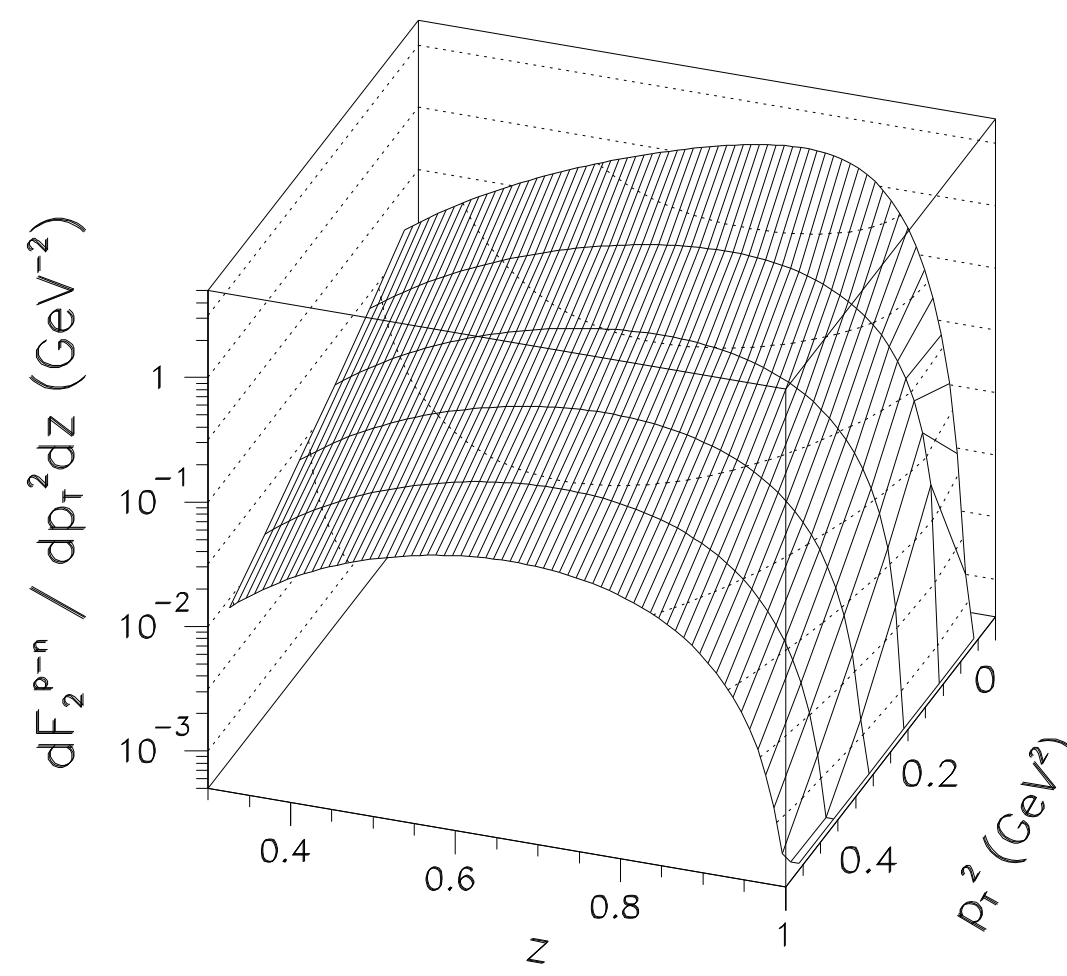

Figure 3: The neutron electroproduction cross section, corresponding to the pion-pole diagram in fig. 11a, versus $p_{T}^{2}$ and $z$

one, takes into account also the excitation of all resonances decaying to the $\pi n$ channel.

The neutron electroproduction cross section, corresponding to the diagram in fig. 1 $\mathrm{b}$, reads

$$
\begin{aligned}
{\left[\frac{d F_{2}^{p \rightarrow n}\left(x, Q^{2}\right)}{d p_{T}^{2} d z}\right]_{1 b}==} & \frac{1}{\pi^{3}} \int_{\left(m_{N}+m_{\pi}\right)^{2}}^{s_{m}} d s_{1} s_{1} \int_{z}^{z_{m}} d z_{1} \frac{1-z_{1}}{z_{1}^{2}} \times \\
& F_{2}^{\pi}\left(x_{\pi}, Q^{2}\right) \frac{d \sigma_{\pi p}^{c e x}\left(s_{1}, \theta\right)}{d \Omega} \int_{0}^{2 \pi} d \Delta \phi \frac{G_{2}^{2}(t)}{\left(m_{\pi}^{2}-t\right)^{2}} .
\end{aligned}
$$

Here $M_{X}$ is, as before, the effective mass of the jet produced in the $\gamma^{*} \pi$ interaction. The effective mass squared of the $\pi n$ in the final state is $s_{1}$. It ranges from the threshold, $\left(m_{N}+m_{\pi}\right)^{2}$, up to a maximum value $s_{m}$ which we fix at $s_{1}=5 \mathrm{GeV}^{2}$. This choice is dictated by the energy range where reliable data on pion-nucleon scattering exist, and is justified by the fast convergence of the integral over $s_{1}$.

The relative share of the initial proton light-cone momentum carried by the final $\pi n$ 
system, $z_{1}$, ranges from $z$ up to $z_{m}$, which is

$$
z_{m}=\min \left\{\frac{z s_{1}}{m_{N}^{2}} ; 1\right\}
$$

The value of $z_{1}$ controls the c.m. energy of the photon-pion collision, $M_{X}^{2}=s\left(1-z_{1}\right)$. $d \sigma_{\pi p}^{c e x}\left(s_{1}, \theta\right) / d \Omega$ is the differential cross section of the pion-proton charge-exchange scattering, where $\Omega(\theta, \phi)$ is the c.m. solid scattering angle. The polar angle is related to other variables as

$$
\cos \theta=1-\frac{2 s_{1}}{s_{1}-m_{N}^{2}}\left(1-\frac{z}{z_{1}}\right)
$$

The four-momentum squared of the virtual pion in this case reads

$$
-t=\frac{1}{z_{1}}\left[\left(1-z_{1}\right)\left(2-z_{1}\right)\left(s_{1}-m_{N}^{2}\right)+\left(1-z_{1}\right)^{2} m_{N}^{2}+q_{T}^{2}\right]
$$

where $\vec{q}_{T}$ is the transverse momentum transfer by the pion exchange,

$$
q_{T}^{2}=p_{T}^{2}+k_{T}^{2}-2 p_{T} k_{T} \cos (\Delta \phi)
$$

and $\vec{k}_{T}$ is the transverse momentum of the neutron relative to the total momentum of the $\pi n$ system,

$$
k_{T}=\frac{s_{1}-m_{N}^{2}}{2 \sqrt{s_{1}}} \sin \theta .
$$

The angle $\Delta \phi$ is the azimuthal angles between $\vec{q}_{T}$ and $\vec{k}_{T}$ of $\gamma^{*} p \rightarrow X(\pi n)$ and $\pi p \rightarrow \pi n$ scatterings.

The formfactor $F_{2}(t)=\exp \left[R_{2}^{2}\left(t-m_{\pi}^{2}\right)\right]$ effectively takes into account the dependence of the $\pi p$ charge-exchange amplitude and the virtual pion photoabsorption cross section on the off-shellness of the pion. The parameter $R_{2}^{2} \approx 0.6 \mathrm{GeV}^{-2}$ was fitted in [1, 13] to describe the data on pion production in $N N$ and $\pi N$ interactions.

The charge-exchange amplitudes, $\pi^{0} p \rightarrow \pi^{+} n$ and $\pi^{-} p \rightarrow \pi^{0} n$ (both are included in eq. (5)) were calculated using the code generating the spin- and isospin-amplitudes for pionnucleon scattering, which was kindly rendered to us by Prof. R.A. Arndt. The code is based 
on the results of recent phase-shift analyses [7] of available experimental data on elastic and charge-exchange pion-nucleon scattering. We used $s_{m}=5 \mathrm{GeV}^{2}$ as an upper limit of the integration over $s_{1}$ in eq. (5). Most of the data used in the analyses [7] are within this energy range, and we checked that the cross section eq. (5) well saturates at this value of $s_{m}$.

The results for the contribution of the diagram in fig. 1 $1 \mathrm{~b}$ are depicted in fig. 1 . This

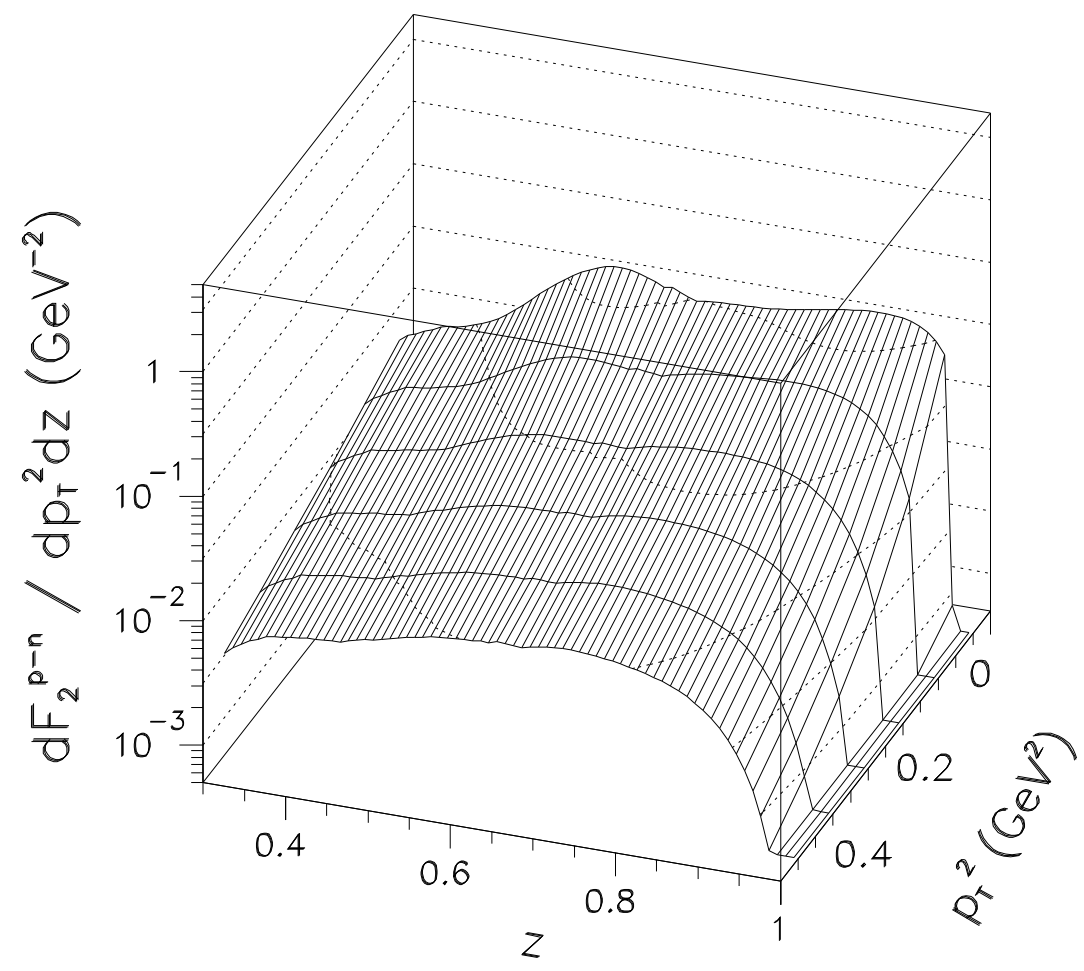

Figure 4: The neutron electroproduction cross section, corresponding to the pion-pole diagram in fig. 1 $\mathrm{b}$, versus $p_{T}^{2}$ and $z$.

contribution to the cross section of electroproduction of the neutron turns out to be about an order of magnitude smaller than that for the diagram in fig. Пa. However, the latter steeply decreases with the transverse momentum of the neutron, and the role of this background increases. We study this problem in more detail below.

Note that the $\pi N$ charge-exchange amplitude presented in the diagram in fig. 마 may be treated as a result of $\rho$-Reggeon exchange, provided that the c.m. energy squared $s_{1}$ is sufficiently high. This is not the case, since we restricted $s_{1} \leq 5 \mathrm{GeV}^{2}$, but for higher values of $s_{1}$ we refer to the next section. 


\section{The Reggeon-exchange contribution (fig. 2a)}

As was explained in the Section 2, in order to bring the pion closer to its mass shell, one has to increase the rapidity gap, however, one faces the problem of the increasing background from the leading Reggeon exchange. Those are $\rho$ and $a_{2}$ Reggeons as is demonstrated in fig. 2a. In this section we make an estimate of this contribution.

The contribution of the mechanism of neutron production shown in fig. fa can be represented in the form

$$
\left[\frac{d F_{2}^{p \rightarrow n}\left(x, Q^{2}\right)}{d p_{T}^{2} d z}\right]_{2 a}=\frac{2 g}{16 \pi^{2}}\left[g_{\rho}^{2} G_{\rho}^{2}(t)+g_{\omega}^{2} G_{\omega}^{2}(t)\right](1-z)^{2 \alpha_{R}^{\prime}|t|}\left|\eta_{R}\right|^{2} F_{2}^{R}\left(x_{R}, Q^{2}\right),
$$

where $\alpha_{R}^{\prime} \approx 0.9 \mathrm{GeV}^{-2}$ is the universal slope of the Regge trajectories.

We see that that the $(1-z)$-dependence of eq. (11) is less steep than that of the pion pole contribution given by eq. (5). This is because $\rho$ and $a_{2}$ Regge trajectories have higher intercepts than the pion, $\alpha_{\rho}(0) \approx \alpha_{a_{2}}(0) \approx 1 / 2$.

We neglect the $t$-dependence of the signature factor $\eta_{R}(t)$ and fix it at $t=0, \eta_{\rho, a_{2}}(0)=$ $\mp i+1$.

We use the couplings $g_{R}$ and the formfactors $G_{R}(t)=\exp \left(R_{R}^{2} t\right)$ as they were fixed by the Regge fit [17] to available experimental data on high-energy hadronic interactions, $g_{\rho}^{2} / 4 \pi=0.18 \mathrm{GeV}^{-2}, g_{a_{2}}^{2} / 4 \pi=0.4 \mathrm{GeV}^{-2}, R_{\rho}^{2}=2 \mathrm{GeV}^{-2}, R_{a_{2}}^{2}=1 \mathrm{GeV}^{-2}$. We assume that $F_{2}^{R}=F_{2}^{\pi}$ and $x_{R}=x_{\pi}$. The cross section, calculated with eq. (11) and these parameters is depicted in fig. 0 as a function of $z$ and $t$.

Note that the $a_{2}$-Reggeon contribution, which is about the same as from the $\rho$-Reggeon, was missed in ref. 四.

\section{Diffractive electroproduction of neutrons (fig. 2b)}

In the case of double diffractive dissociation the proton may produce a jet containing the neutron, for instance like it is shown in fig. $2 \mathrm{~b}$. As different from the single-pion exchange in fig. 1]b, this contribution is rapidity-gap independent, or can even grow with $\Delta y$ [18]. Thus, one may expect a substantial correction from this process. 


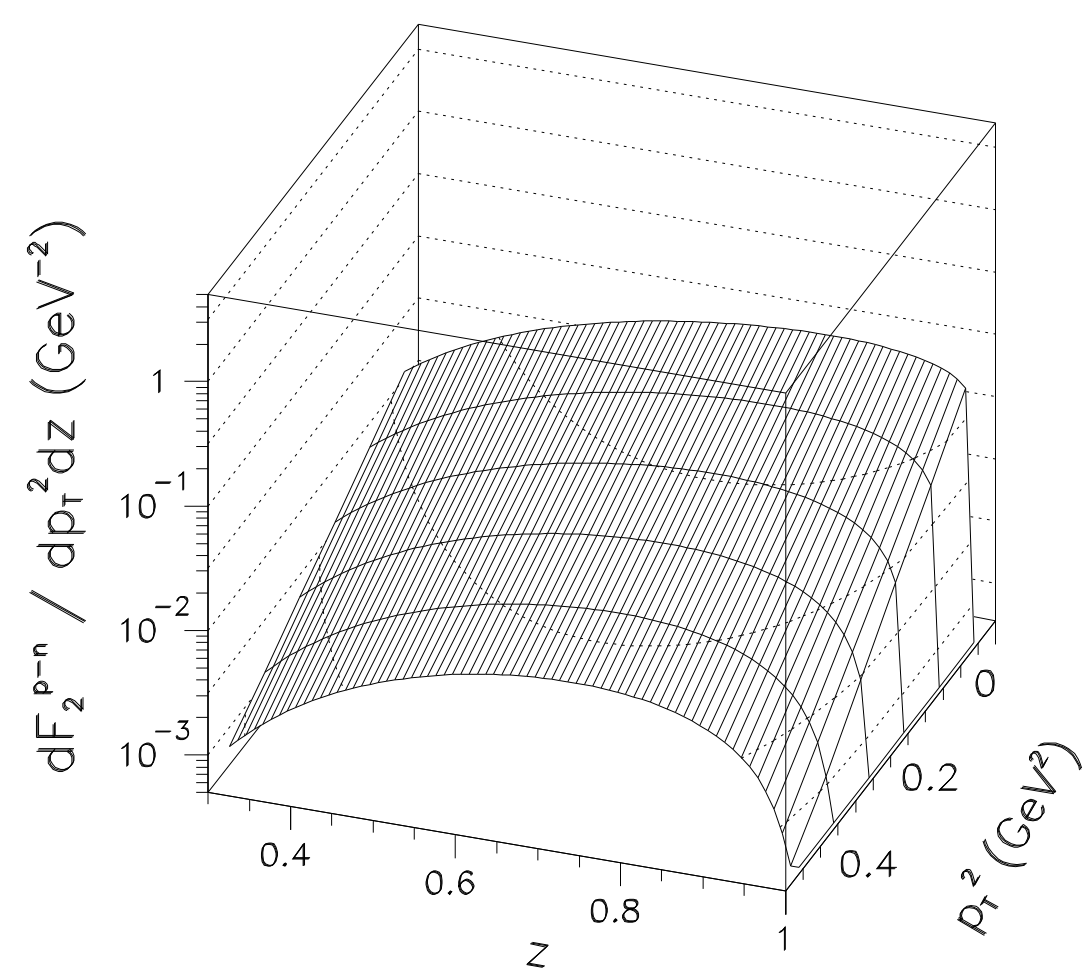

Figure 5: The neutron electroproduction cross section, corresponding to the Reggeon-exchange diagram in fig. 2aa, versus $p_{T}^{2}$ and $z$.

At $z \rightarrow 1$ the reaction in fig. 2 $\mathrm{b}$ is known to be well described by the so called Deck mechanism [19] shown by the diagram in fig. 6.

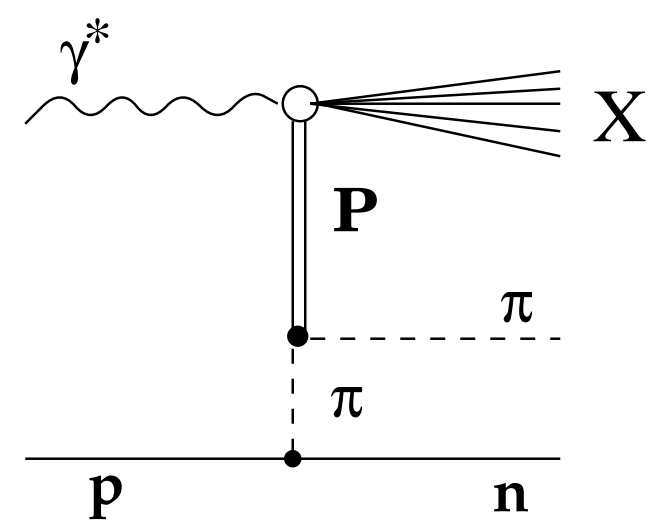

Figure 6: The Deck mechanism of the double diffractive dissociation electroproduction of the neutron.

This mechanism corresponds to the diffractive dissociation of the photon on a pion and 
can be treated as a single-diffraction part of the process shown in fig. 1a. The relative contribution of the single photon diffraction to the total photoabsorption cross section on a pion is expected to be about $2 / 3$ of that in $\gamma^{*} p$ interaction if one uses the approximate Pomeron factorization. The photon diffraction on a proton was measured recently at HERA [18] at about $10 \%$ of the total photoabsorption cross section. Thus, we expect about $6 \%$ correction to the measured pion structure function coming from the diffractive mechanism depicted in fig. 6. On the other hand, even the proton structure function is known only up to such a correction, so we neglect it.

\section{Discussion and conclusions}

The contributions of the diagrams in figs. 1 $1 \mathrm{a}, \mathrm{b}$ and $2 \mathrm{a}$, presented in figs. 355 show that the first one, fig. 11a dominates by about an order of magnitude at small $p_{T}^{2} \leq 0.3 \mathrm{GeV}^{2}$. This is demonstrated also on the $z$-distribution at $p_{T}^{2}=0$ in fig. 7 and on $p_{T}^{2}$-dependence in fig. \&.

We should comment, however, on the reliability of our predictions. In the process under discussion one measures the structure function of the virtual pion, so a problem arises concerning the extrapolation of the result to the pion pole. There are quite a few theoretical schemes known in the literature, which treat the off-mass-shell pion in different ways.

The approach based on the dispersion relations over the pion four-momentum squared treats the pion pole explicitly, but one gets in trouble trying to include many other singularities, $3 \pi$-cut, $\pi^{\prime}$-pole etc.

Using Feynman diagrams one takes into account off-shellness of the pion by means of the vertex function $G_{1}(t)$ in eq. (葘). This formfactor is supposed to incorporate effectively other singularities in the dispersion relation. However, an additional $z$-dependence of the formfactor is possible because some of the further singularities in the dispersion relation correspond to particles of higher spin, which results in a different energy-, or $z$-dependence $\left(1-z=M_{X}^{2} / 2 m_{N} \nu\right)$ of the interaction amplitude compared to that for the pion pole. Usually the modification of $z$-dependence is neglected in the approach based on Feynman diagrams [2].

In order to incorporate with different energy dependences corresponding to the exchange 


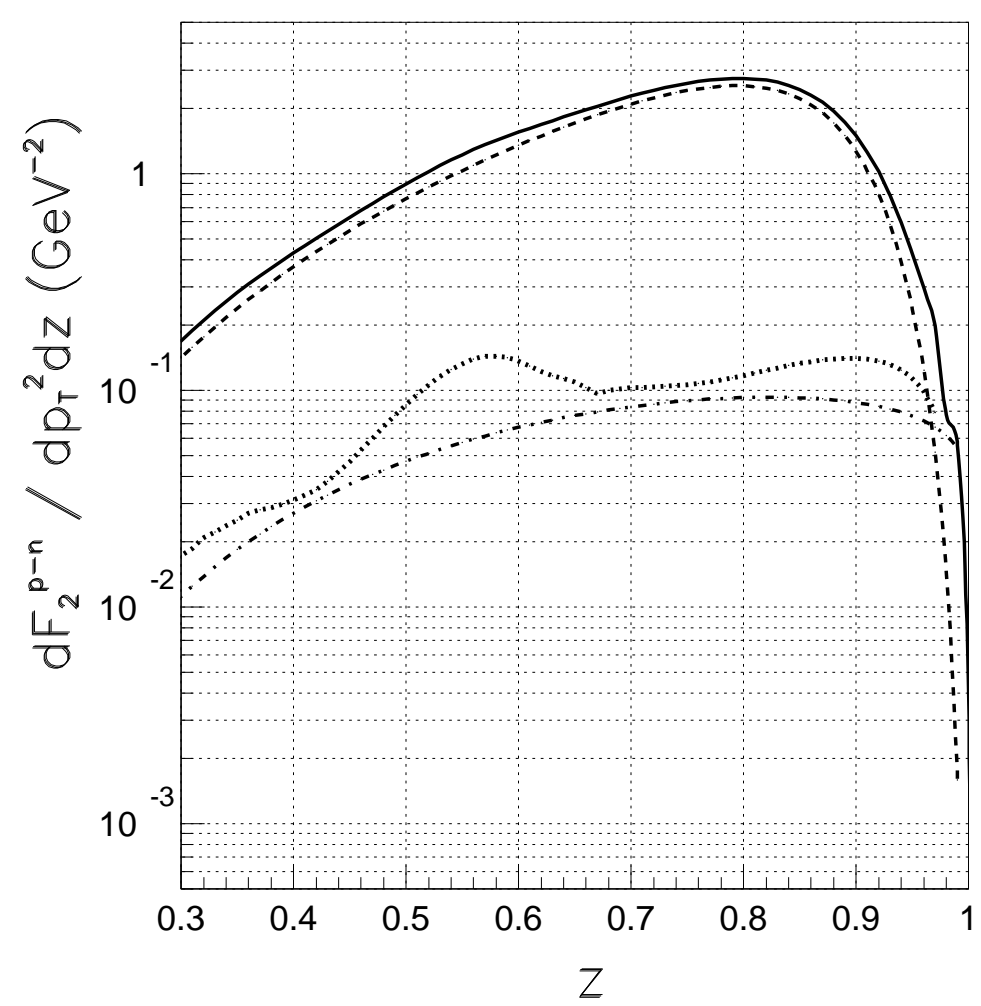

Figure 7: Comparative contributions of the diagrams in fig. 1a (dashed curve), fig. 1b (dotted curve) and fig. 2a (dot-dashed curve) to the neutron electroproduction cross section as a function of $z$ at $p_{T}^{2}=0$. The sum of the three mechanisms is depicted by the solid line.

of states with different spins the Regge scheme was suggested many years ago, which turned out to be extremely successful describing the energy dependence for many hadronic reactions. The main idea is to use a more appropriate variable, rather than $t$, for the dispersion approach, which is the complex angular momentum. Following [5, [] we use the Reggeized

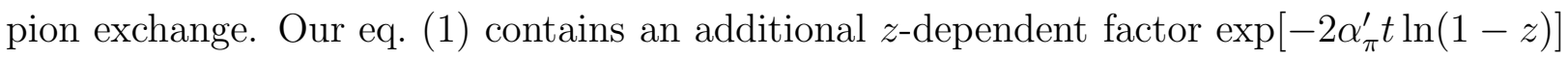
compared with the Feynman diagram. This factor is of special importance at small $(1-z)$ or moderately large $t$. The wide-spread opinion that Reggeization is not important for the pion, since $\alpha_{\pi}(0) \approx 0$, is true only at $t=0$.

A $z$-dependence of the cross section, different from the standard Regge approach was suggested in [4] (and references therein). It is based on the light-cone decomposition of the nucleon over the Fock components containing extra pions. Although the factorization is not 

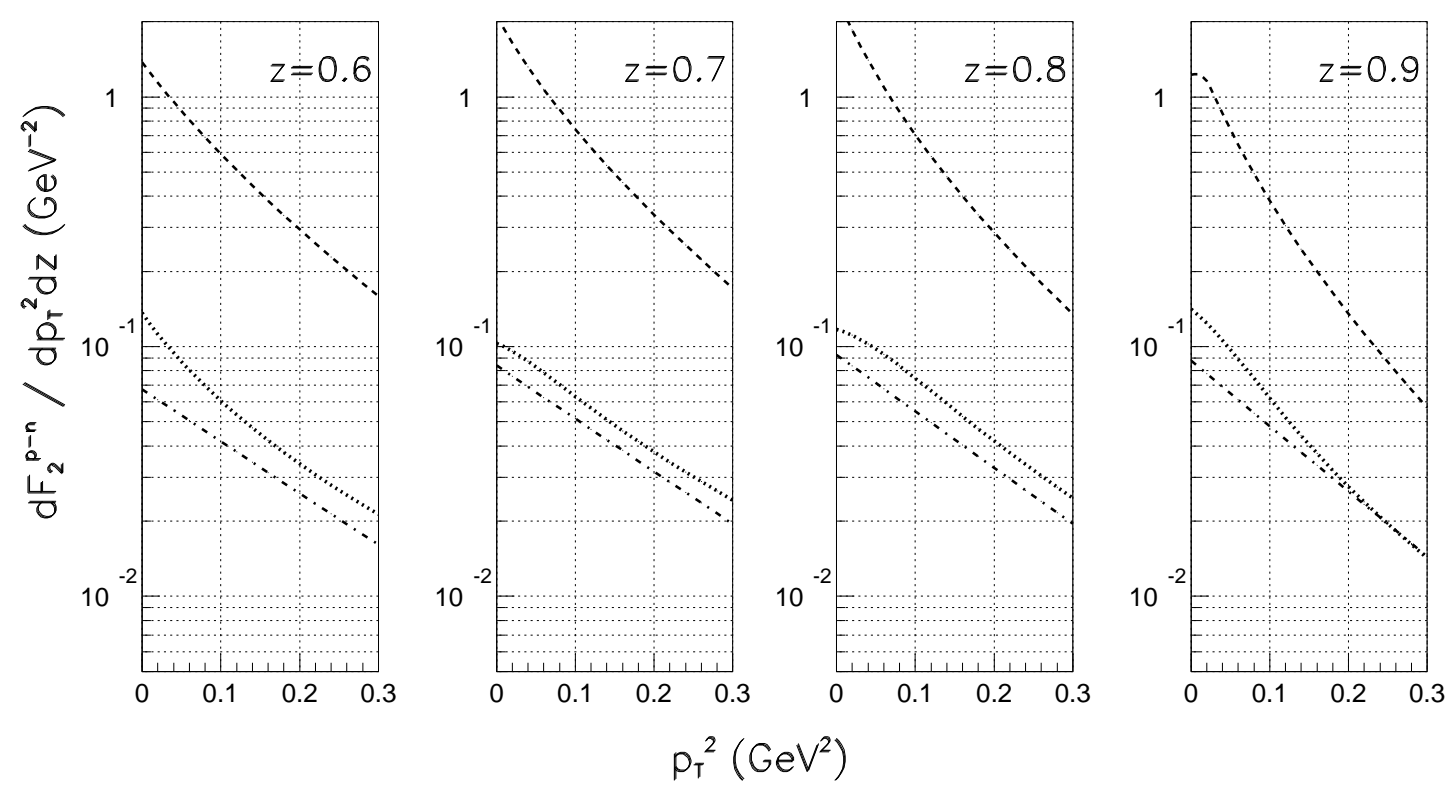

Figure 8: $p_{T}^{2}$-distribution of the cross sections of electroproduction of neutron corresponding to the diagrams in fig. 1a (dashed curve) fig. 1b (dotted curve) and fig. 2a (dashed-dotted curve).

proved for such a decomposition, it might be a reasonable approximation. Neglecting the components with two or more pions one comes to the conclusion [12] that a natural variable for the formfactor for the off-shell pion is the effective mass squared of the pion-nucleon fluctuation, which is equivalent to $t /(1-z)$. This results in a $z$-dependence very different from the one dictated by Regge theory, and in an unusual $t$-dependence which becomes very steep towards $z=1$. Although it was claimed in [4] that such an approach cannot be used at high $z$ where Reggeization of the pion is important, the main results of [4] are obtained in the kinematical region $z>0.7$, which is known to be a domain of triple-Regge phenomenology. What is especially important, theoretical predictions are reliable only at

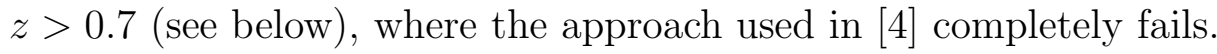

The $\rho$-meson exchange is treated in [4] on the same footing. The failure of such an approach can be easily demonstrated on a $\rho$-dominated reaction, for instance, $\pi^{-} p \rightarrow \pi^{0} n$, which is well known to be governed by the $\rho$-Reggeon exchange. Besides, the $a_{2}$-exchange, neglected in [4] is as important in the diagram in fig. 2aa, as the $\rho$, due to exchange degeneracy.

The distribution over $z$ can be reliably predicted only at high $z \geq 0.7$. Indeed, the 
$z$-dependence of the contribution of the diagram in fig. 17a is controlled by the energy dependence corresponding to the Reggeized pion exchange, which we know quite well. However, the left-side slope of the peak in $z$-distribution in fig. 7 is only due to the growth of $t$ with decreasing $z$ in accordance with eq. (位). As a result, the cross section falls down due to the pion propagator and the formfactor $G_{1}^{2}(t)$ in eq. (目). The latter, as was commented above, is poorly known, and the results presented in fig. 7 correspond to an optimistic case of small radius $R_{1}^{2}=0.3 / \mathrm{GeV}^{-2}$. Nevertheless, even with a large radius $R_{1}^{2}=2 \mathrm{GeV}^{-2}$, the pion pole contribution dominates by an order of magnitude at $z \approx 0.7-0.8$. To be safe one should study the pion-pole contribution in this kinematical region.

The $p_{T}^{2}$-dependence may be substantially affected by the uncertainty in the slope of the formfactor $G_{1}(t)$ mentioned above. The results corresponding to the choice of a small radius are depicted in fig. 8 and demonstrate the dominance of the pion pole. However, at large radius $R_{1}^{2}=2 \mathrm{GeV}^{-2}$ the relative contribution of the pion pole fig. 17a steeply decreases with $p_{T}^{2}$. To be safe one should restrict $p_{T}^{2}<0.2-0.3 \mathrm{GeV}^{2}$.

One can conclude from present analyses that the pion-pole contribution to the deepinelastic cross section of the leading neutron production can be reliably determined, and the pion structure function at small $x_{\pi}$ can be well measured. However, in order to disentangle the pion pole contribution (fig. 1a) and the background one should select the events around the maximum in $z$-distribution shown in fig. [1. Dependent on the statistics, one can choose an interval $z \approx 0.7-0.9$ and $p_{T}^{2}<0.2-0.3$.

Summarising, the important signature of the contribution of the pion pole graph in fig. 11a is the steep decrease of the cross section towards $z=1$ at $z>0.8$ as is shown in fig. (7) This is mainly due to the spinelessness of the pion, and our predictions in this kinematical region are least affected by theoretical uncertainties, particularly those in the pion-nucleon formfactor.

Another signature of the pion-pole, the steep $p_{T}^{2}$ dependence of the cross section, can be smeared due to the smallness of the radius in the pion-nucleon formfactor. In the $z$-interval of the dominance of the pion-pole demonstrated in fig. 7 the longitudinal momentum transfer is quite small, and the exchanged pion is not far from the mass shell. For instance, at 
$z=0.9$ the minimal pion virtuality is $|t|_{\min } \approx m_{\pi}^{2} / 2$. This explains why the slope of the $p_{T}^{2}$-distribution depicted in fig. 8 is so large, $B \approx 10 \mathrm{GeV}^{-2}$, in spite of the smallness of the used radius, $R_{1}^{2}=0.3 \mathrm{GeV}^{-2}$. The most part of it, about $7 \mathrm{GeV}^{-2}$, originates from the pion propagator in eq. (1). The rest comes from the $\pi N N$ formfactor and from the Reggeization of the pion pole. The latter part is about the same for the $\rho$ - and $a_{2}$-Reggeons, but the former contribution is much smaller.

An important conclusion is that, regardless of the theoretical uncertainty, the pion pole contribution corresponding to the graph in fig. [1 7 a well dominates by an order of magnitude in the kinematical region $z \approx 0.7-0.9$ and $p_{T}^{2} \leq 0.2-0.3 \mathrm{GeV}^{2}$.

The real challenge to the experimentalists is to identify the pion-pole contribution by measuring the $p_{T}^{2}$ distribution and the $z$-dependence of the produced neutrons for $z \rightarrow 1$ with the required accuracy. Not a simple task with neutrons of several hundred GeV energy.

Acknowledgements: We are grateful to M.G. Ryskin, who participated in the calculations at the early stage, D. Jansen and A. Thomas for the helpful discussions. We appreciate very much the courtesy of Prof. R.A. Arndt, who provided us with the code generating the amplitudes of pion-nucleon interaction, based on the phase-shift analyses [7]. We are thankful to I. Strakovsky, who helped us with running the code, for good comments. We thank H. Holtmann and N.N. Nikolaev for the discussion of the results of ref. [4]. This work was partially supported by INTAS grant 93-0239.

\section{References}

[1] G.G. Arakelyan, K.G. Boreskov and A.B. Kaidalov, Sov. J. Nucl. Phys. 33 (1981) 247

[2] J.D Sullivan, Phys. Rev. D5 (1972) 1732

[3] G. Levman and K. Furutani, ZEUS note 92-107, 1992; preprint DESY 95-142, 1995

[4] H. Holtmann et al., Phys. Lett. B338 (1994) 363

[5] J. Pumplin, Phys. Rev. D8 (1973) 2249

[6] V. Stoks, R. Timmermans, and J. J. de Swart, Phys. Rev. C47 (1993) 512

[7] R.A. Arndt, I.I. Strakovsky, R.L. Workman, and M.M. Pavan, Phys. Rev. C52 (1995) 2120 
[8] L.A. Ponomarev, Sov. J. Part. Nucl. 7 (1976) 70

[9] G.G. Arakelyan and K.G. Boreskov, Sov. J. Nucl. Phys. 30 (1979) 840; 31 (1980) 819

[10] S. Baumgärtner, H.J. Pirner, K. Königsmann and B. Povh, Z. Phys. A353 (1996) 397

[11] W. Koepf, L.L. Frankfurt and M.I. Strikman, preprint TAUP-2298-95, hep$\mathrm{ph} / 9510452$

[12] V.R. Zoller, Z. Phys. C 53 (1992) 443

[13] G.G. Arakelyan and A.A. Grigoryan, Sov. J. Nucl. Phys. 34 (1981) 1338

[14] J.F. Gunion and D.E. Soper, Phys. Rev. D15(1977) 2617

[15] B.Z. Kopeliovich and B. Povh, preprint MPIH-V12-1995, to be published in Phys. Lett. B

[16] The H1 Collaboration, T. Ahmed et al., Nucl. Phys. B439 (1995) 471

[17] P.E. Volkovitskii et al., Sov. J. Nucl. Phys. 24 (1976) 648

[18] The H1 Collaboration, T. Ahmed et al., Phys. Lett. B348 (1995) 681

[19] S.D. Drell and K. Hiida, Phys. Rev. Lett. 7 (1961) 199; R.T. Deck, ibid. 13(1964) 169 